\title{
On reference modelling of integrated manufacturing systems using OOA
}

\author{
G.Vosniakos* and M.Williams** \\ * National Technical University of Athens, Dpt of Mech. Engng, \\ Patission 42, GR-10686, Athens, Greece, tel +301 7723691, \\ fax+301 7723689, e-mail Vosniakos@central.ntua.gr \\ ** UMIST, Dpt of Mechanical Engineering, PO Box 88, Manchester \\ M60 1QD, Britain, tel +44161 2003800, fax +44161 2003803, \\ e-mailmcjicmaw@fsl.me.umist.ac.uk
}

\begin{abstract}
This work advocates the use of Object Orientation as basis of a methodology for creating reference models of manufacturing systems. Work on reference modelling of manufacturing systems integration is reviewed first. Then this work's own standpoint on reference modelling is outlined with definition of the main concepts. Reference models are distinguished from actual-implementation models through the need for generalisation, abstraction and expansion ability. Coad and Yourdon's Object Oriented Analysis is used to demonstrate reference modelling of structural, transformation and procedural aspects of a batchprocessing shop-floor system, explaining how manufacturing systems entities are mapped to OOA entities. The main concepts introduced along with discussion of relevant examples are a phenomenological View, application-related Views, abstraction hierarchy of objects and expansion ability of the model to advance from abstract to environment-specific reference models. Modular instancing of entities is also discussed in the context of further work.
\end{abstract}

Keywords

System Analysis, Manufacturing Systems, Shop-floor, Coad-Yourdon's OOA.

\section{INTRODUCTION}

Manufacturing system integration typically addresses many subsystems performing complex sequences of actions, which possibly run concurrently. Cross-area integration in manufacturing systems refers to the specification of common goals, outputs from one area forming inputs to other areas, crossarea consultation between functions, and repercussions of parameter changes in one area onto other areas (Burbidge, 1987). 
In an effort to design and implement integrated manufacturing systems the concept of reference models has been explicitly or implicitly recognised to be of fundamental importance (Biemans, 1991),(Jochem, 1989). In general, the term 'reference model' describes an 'ideal' or 'target' configuration of a manufacturing system or of parts of it and therefore it has a 'prescription' or 'recipe' connotation. A reference architecture by contrast defines a modelling methodology (rules referring both to the modelled domain and to the modelling task and structures, possibly including a modelling language) (Aguiar, 1995). Another term which is often used in literature as equivalent to a reference model of a high level of abstraction is 'generic model', e.g. in a production control context (Filip, 1993).

According to one classification, there are four main types of use of reference models for manufacturing systems: Benchmarking, Diagnosis, Design, Evaluation (Bohms, 1993).

A CIM Reference model in the context of process industries is given in (McCarthy, 1990). It consists of a number of sub-models, namely organisational, control strategy, information flow and network connectivity used to derive the functions needed in a plant information system

In designing automated production control systems, system aspects captured by a reference model are categorised as behavioural, information and functional (Lhoste, 1993). A 'diachronic' system life cycle is advocated, using the equivalent of 'invariant' libraries of standard and re-usable behaviour, information and functions.

Taxonomies form a good baseline for the development of reference models, one obvious thing they lack being functional interactions of the categorised objects. A taxonomy example referring to materials handling environments (Krni, 1995) can be easily paralleled to an 'uninstantiated' reference model. Another six-level taxonomy of typical CIM activities is the basis for planning the Information Systems in CIM (Camarinha-Matos, 1994).

Research needs with respect to CIM architectures defined in (Aguiar, 1995) follow four streams: extension of CIM-OSA, development of object -oriented modelling tools, development of standard infrastructural type of tools and building information modelling and manipulation tools. The need is recognised for populating a system engineering workbench with a library of reference models that encapsulate knowledge of user requirements associated with resource capability and of information technology solutions based on enterprise domain typification.

\section{OBJECT ORIENTED ANALYSIS}

The methodology chosen in this work to create reference models of (integrated) manufacturing systems is Object Oriented Analysis (OOA) by Coad and Yourdon (Coad, 1991). An OOA model can be derived in five separate modelling steps : finding Classes and Objects, identifying Structures (whole-part, genspec, instance connections and event-remembered objects), identifying Subjects, defining Attributes, defining Services.

Manufacturing system reference models need to reflect the Structural, Transformation and Procedural definition of systems. Structural definition is the description of all physical and conceptual elements of the system. Transformation definition is the description of the functions performed by the system elements. Procedural definition is the description of time-phased interactions of system elements, i.e. the dynamic behaviour of the system (Hitomi, 1990).

The transformation definition of the system in OOA is given through services indicating what behaviour will be provided by an object within a class. The procedural definition of the system, i.e. communication and sequencing requirements, in OOA involves defining communication messages between objects. These messages invoke a response such as replying to the original object, displaying results, monitoring a dynamic attribute etc. (Coad, 1991).

Before demonstrating the adequacy of OOA for creating reference models of manufacturing systems it is appropriate to highlight the distinguishing characteristics of reference models, as these stem from the 


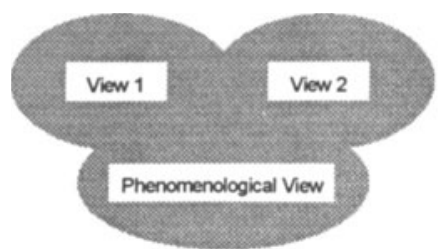

\begin{tabular}{|c|c|c|c|c|c|}
\hline OBNECT 1 & View & Instance Connection & View & OBNECT 2 & View \\
\hline \multirow{2}{*}{$\begin{array}{l}\text { Attribute_1.1 } \\
\text { Attribute_1.2 }\end{array}$} & \multirow{2}{*}{$\begin{array}{l}P \\
P\end{array}$} & ic_1 & $P$ & \multirow{2}{*}{$\begin{array}{l}\text { Attribute_2.1 } \\
\text { Attribute_2.2 }\end{array}$} & \multirow{2}{*}{$\begin{array}{l}P, 2 \\
1,2\end{array}$} \\
\hline & & & & & \\
\hline Service_1.1 & \multirow{2}{*}{1} & Message Connection & View & \multirow{2}{*}{$\begin{array}{l}\text { Service_2.1 } \\
\text { Service_2.2 }\end{array}$} & \multirow{2}{*}{$\begin{array}{l}1 \\
2\end{array}$} \\
\hline (n) & & $m c \_3=f\left(s \_1.2, s \_2.1\right)$ & 1 & & \\
\hline
\end{tabular}

Figure 1 Reference model Views and Objects and their communication.

requirements for generalisation, abstraction and expansion ability.

\section{REFERENCE MODEL CONCEPTS}

Generalisation ability is required in order to cover as many possible implementations of the proposed reference model as possible. Abstraction ability is required in order to modularise the model according to a variety of changing criteria. Expansion ability is needed in order to move from an abstract to a more concrete and finally to an implementation version of the system. Note that the term 'implementation' of a reference model is used as synonymous to realisation of a physical or software system corresponding to that model.

\section{Views}

In this work, a reference model is considered to support one or more 'Views' each of which is tied to a particular application. This is rather similar to the concept of external schemata in a database model. A phenomenological view is considered to form the basic reference model of any manufacturing system. This view describes how the system appears to be working to an external observer. Typically, its objects, attributes and services are generic enough to be applicable to a variety of circumstances. Therefore this view cannot be concerned with details of complex algorithms such as planning and scheduling. Any other view of the manufacturing system essentially extends or abstracts certain areas of the phenomenological view.

Views may share objects and some of their attributes, services, messages and instance connections, in which case these objects, attributes etc. are termed common. Figure 1 illustrates this notion. Object_1 is common to views $P$ and 1 , whereas Object_ 2 is common to views $P, 1$ and 2 . Instance connection ic_1 belongs to view $P$, which means that it refers to aspects (attributes and services) of Objects 1 and 2 that pertain to View P. Message connection mc_3 refers to two services pertaining to view 1, hence it belongs to view 1 , too. Views may also possess objects and some of their attributes, services etc. exclusively, in which case these objects, attributes etc. are termed private.

\section{Abstraction hierarchy}

For any specific View (application) a hierarchy of reference models may be defined, starting at the top with a model generic enough to be both configurable to individual company environments and reusable in different situations. Subsequent levels in the hierarchy, corresponding to different levels of abstraction, would add progressively more detail to the top level model, leading towards specific implementations. This is possible according to the principle of inheritance where the more specific class inherits the characteristics of the more generic ones. In addition, relationships can be defined between classes that belong to the same level of abstraction, thus becoming part of a model extension, see Figure 2. 


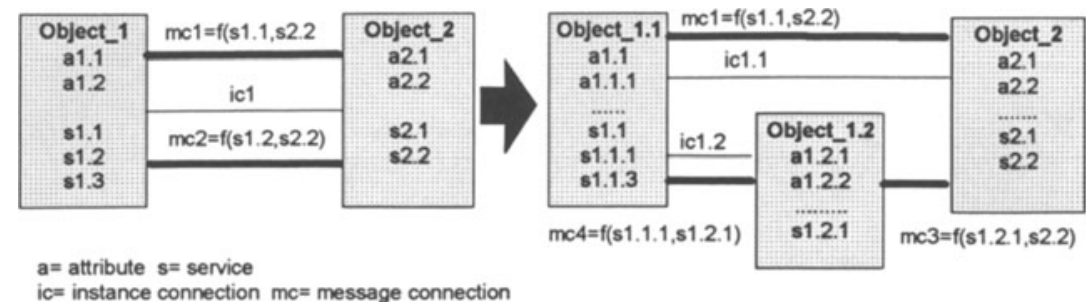

Figure 2 Modular expansion concept.

\section{Expansion ability}

In order to carry out an expansion of the model a set of transformation rules is needed referring to classes, attributes, services, instance connections and message connections. Model expansion takes two forms : adding classes / objects to a new level in a generalisation-specialisation structure and adding new classes independently of any existing structure.

In the first case the class / object inherits attributes and services of the level above. Any new attributes and services will be simply added. Instance connections and message connections will also be added to reflect the behaviour of the new class / object.

In the second case the new object will in most cases define in further detail some aspect of an existing object, hence the initial object can be considered to be split into at least two new objects. These will be related by instance connections and possibly by message connections, too. The instance and message connections of the initial object will have to be preserved in meaning, but will probably change in form, see Figure 2. If a service of the original object which is involved in a message connection is retained in the new picture, then the message connection is most probably to be retained, too. If, on the other hand, the service is transformed into separate services, these will probably have to communicate both with each other and directly or indirectly with the objects to which the initial message connection referred. The above depend very much on the nature of services, of the instance connections and of the message connections, hence they are not cast in stone.

\section{Language}

A reference model should be unambiguous, therefore it has to be expressed in a particular formal language. If this language is computer parsable, then the reference model can also be executable, i.e. be possible to 'interpret' by computer in order to generate software that would, at least partly, implement the relevant Views (applications). Coad-Yourdon's OOA is the modelling language used in this work as illustrated through the example that follows.

\section{A SHOP-FLOOR REFERENCE MODEL FOR BATCH PRODUCTION}

Due to the size even of a part of a manufacturing system and the many possibilities for different applications pertaining to any such sub-system, only the phenomenological view has been concentrated on in this work. The sub-system modelled was the 'production execution' on the batch-processing shop floor for mechanical components, mainly because of its relevance of the authors background. In a later expansion phase additional system responsibilities can be added. The main features of the reference model developed follows.

\section{Subjects, classes, objects and attributes}

Four subjects were identified : Shop-floor, Product, Inventory and Personnel, see Figure 3. The shop- 


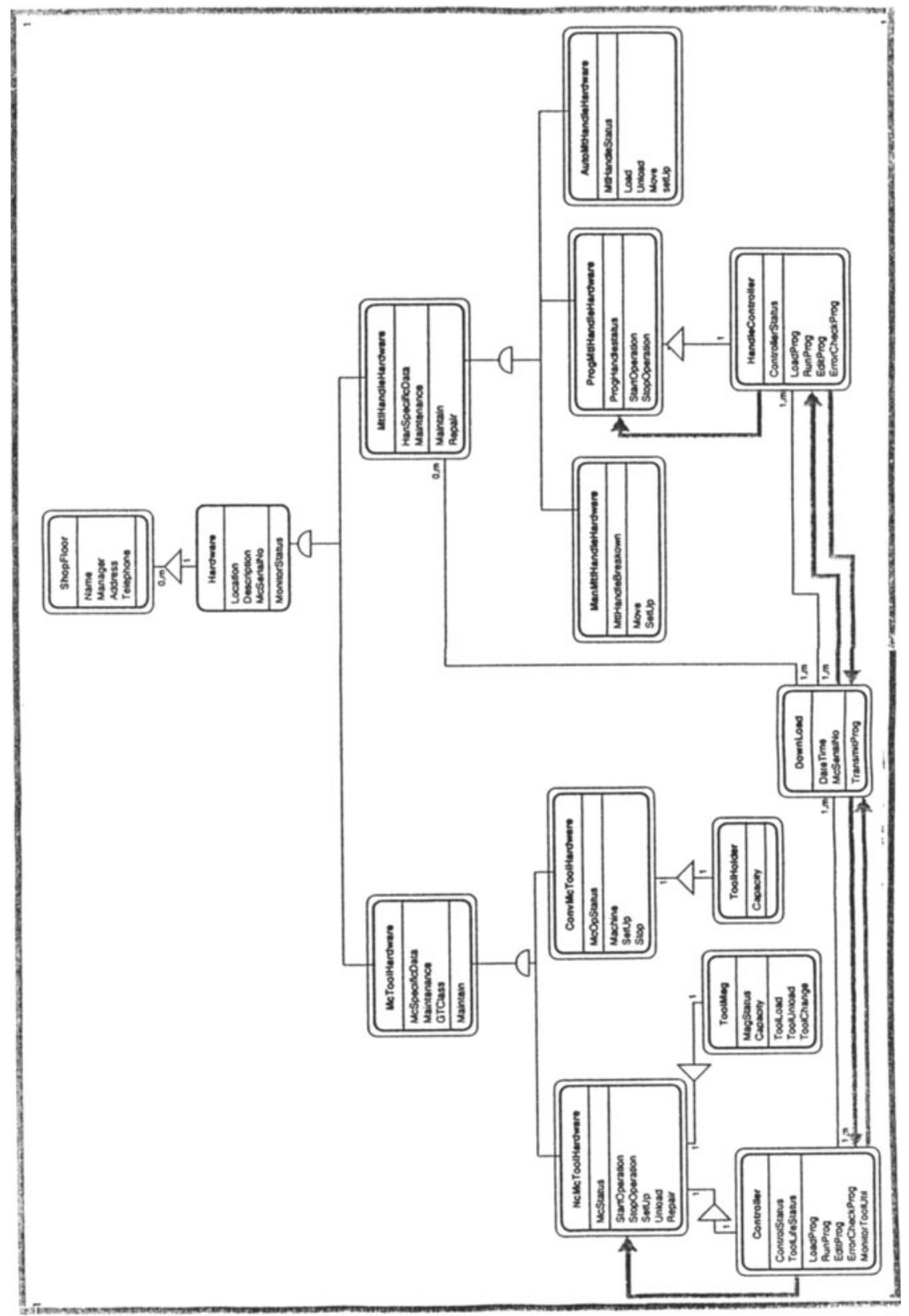

Fig. 3(a) Reference model for subject Shop floor 
Part Two Modelling of Products, Processes and Systems

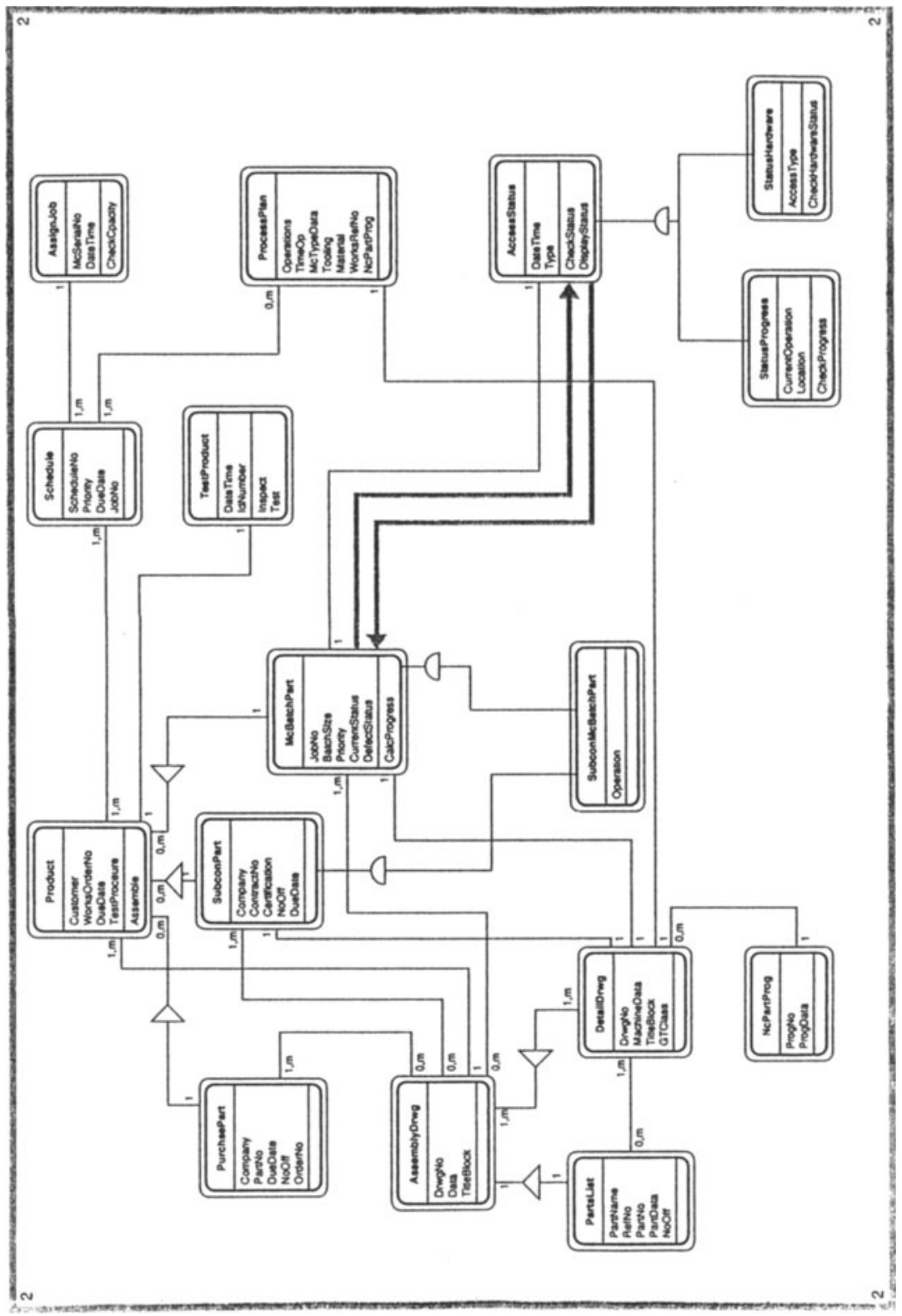

Fig. 3(b) Reference model for subject Product 

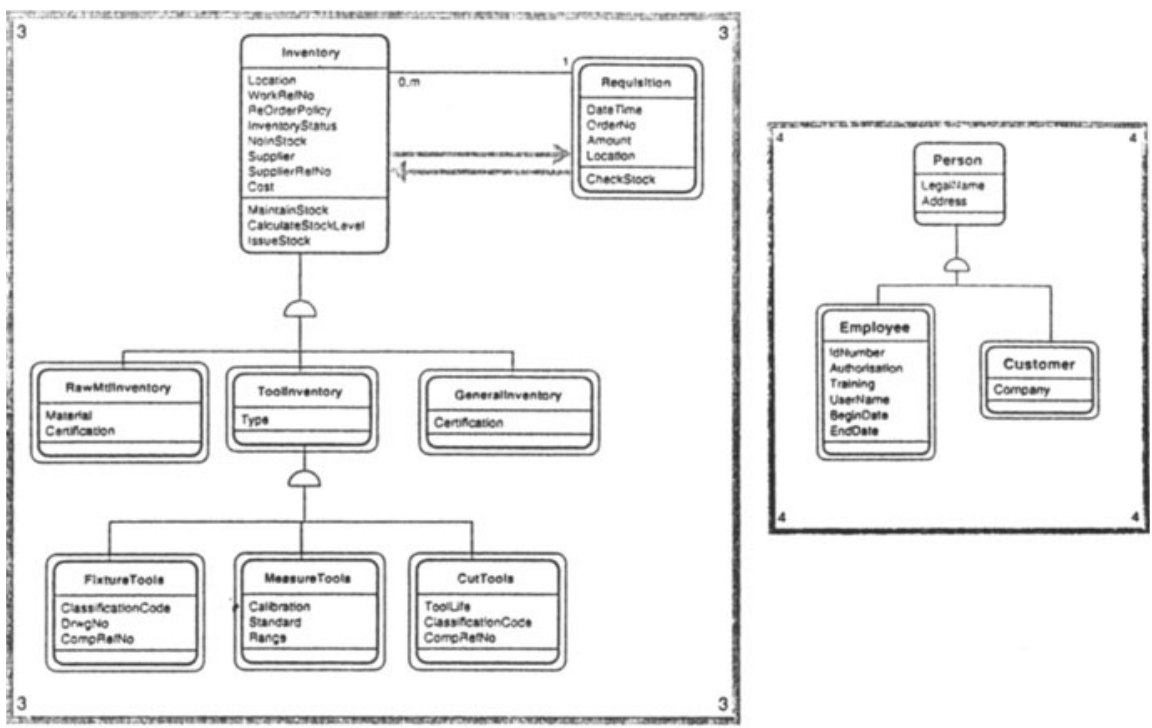

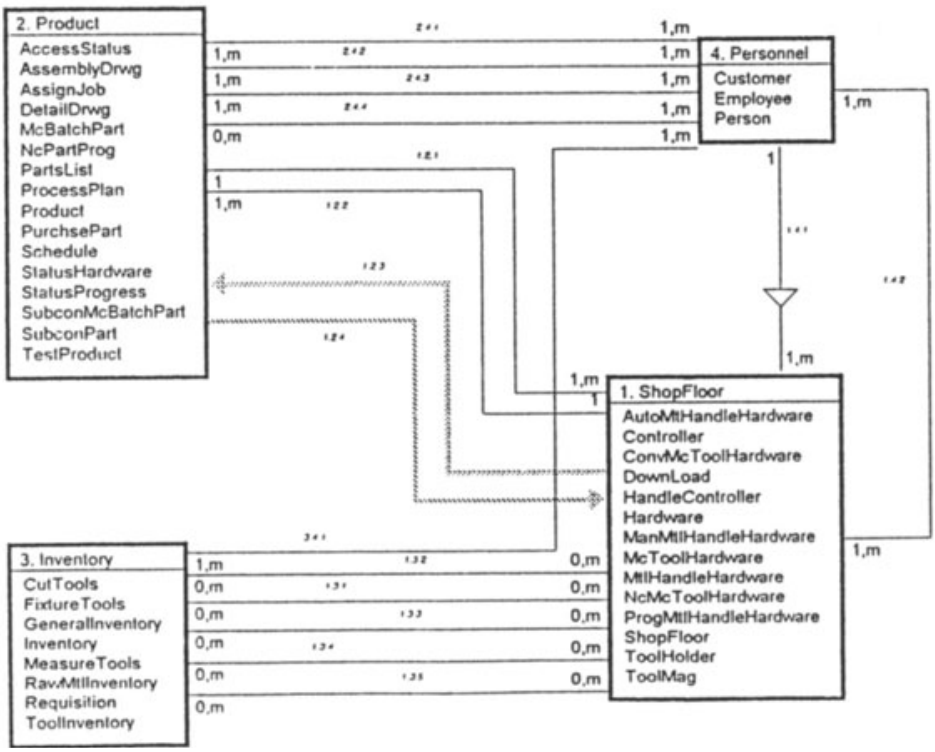

Fig. 3 (c) Reference model for subject Inventory

(d) Reference model for subject Personnel

(e) Subject connections - see also Appendix 
Table 1 Instance and message connections across subjects - supplement to Figure 3(e)

\begin{tabular}{lcllll} 
Index & c & From - to & Index & c & From - to \\
\hline 1.2 .1 & ic & AccessStatus-Hardware & 1.4 .1 & ic & Employee-Shop Floor (part of) \\
1.2 .2 & ic & NcPartProg-DownLoad & 1.4 .2 & ic & Employee - Hardware \\
1.2 .3 & mc & AccessStatus-Hardware & & & \\
1.2 .4 & mc & Hardware-AccessStatus & 2.4 .1 & ic & McBatchPart-Employee \\
\hline 1.3 .1 & ic & CutTool - ToolMag & 2.4 .2 & ic & AccessStatus- Employee \\
1.3 .2 & ic & CutTools - ToolHolder & 2.4 .3 & ic & TestProduct - Employee \\
1.3 .3 & ic & MeasureTools - ToolMag & 2.4 .4 & ic & AssignJob - Employee \\
1.3 .4 & ic & FixtureTool -McToolHardware & & & \\
1.3 .5 & ic & Inventory-MtlHandleHardware & 3.4 .1 & ic & Requisition - Employee \\
\hline
\end{tabular}

floor subject includes a large Gen-Spec structure called Hardware, see Fig 3(a). All its instances have a location, description and identity inherited from the Hardware class. Machine tools are described by their capabilities, maintenance related data and Group Technology coded operations available. As a further specialisation there are $\mathrm{NC}$, conventional and material handling machines. They possess, among other attributes, status, capability specifications and maintenance data.

In the Product subject, see Figure 3(b), the class Product has customer, works order number, due date and test procedure attributes, the latter emphasising the necessity for quality. A product is made up of parts manufactured in house (McBatchPart), purchased from outside (PurchasePart) and subcontracted (SubconPart). McBatchPart has a priority attribute useful for scheduling, a DefectStatus attribute used in Quality Assurance and a current status. Subcontracted parts have a certification attribute emphasising Total Quality. There is also provision for parts partly manufactured in house and partly subcontracted on a per operation basis (McSubconPart). This is connected to SubconPart and McBatch Part in a lattice structure.

Still within the Product subject, AssemblyDrawing is an object representing product structure. It consists of PartsList and of DetailDrawings. There are also objects representing the product structure, namely : PartsList with part name, reference number, part number, part data and number off attributes, and DetailDrawing with drawing number, manufacturing data (tolerances, dimensions, surface finish etc.) and Group Technology codes. There is also a ProcessPlan object used on the shop floor for reference, whose attributes define sequence of operations and machines necessary for production. In addition, a Schedule object is needed for low-level scheduling, with main attributes priority and duedate.

Inventory represents all material resources required by a production system, such as raw materials, general consumables, and tools, see Figure 3(c). Each instance of the inventory class has location, reorder_policy, inventory_status (standby, monitor, order), number_in_stock, supplier and cost attributes. RawMaterial and Generallnventory classes possess Certification attributes. Tools inventory is broken down into cutting tools, fixtures and measuring tools. Cutting tools have a tool life, a classification code detailing dimensions, operations etc. and company reference number, e.g. ISO. Fixtures need a drawing, too, whilst measuring tools are different in that they have a measurement scope, and they need calibration which is carried out according to a standard.

Personnel is the smallest of the subjects, but the corresponding external mapping is detailed, reflecting the importance of human involvement in a manufacturing system, see Figure 3 (d). The Employee attributes include, among other attributes, authorisation level for accessing data and training qualifications.

\section{Instance Connections}

There are several instance connections between objects of the model. A detail drawing, for example, is 
associated with a part machined or subcontracted and also with a process plan (one-to-one relationship), It is also associated with the corresponding parts list and perhaps with an NC program. Note that these are all associations within the same subject.

As an example of instance connections spanning more than one subjects refer to the McBatchPart object. A connection occurs with the ProcessPlan object, as each part has to have a specific process plan produced before it can be manufactured (one-to-one relationship). Additional connections occur with objects AssemDrwg and DetailDrwg, mapping association with the appropriate engineering drawings. Connections also occur with Employee and Hardware. These connections map the association employees and equipment have with a part as it is processed and reflect many-to-many type of relationships, because specific employees and pieces of machinery can process many different manufactured parts and vice versa.

Event-Remembered objects include :

- AssignJob, which relates a schedule and an employee and needs machine identification and datetime attributes

- TestProduct, which relates Product and Employee and has a date-time attribute

- AccessStatus, which relates McBatchPart, Employee and Hardware at a generic level representing the system's capability of retrieving current, up-to-date status information stored in relevant dynamic attributes. This is broken down further into StatusProgress referring to machined batches and StatusHard referring to hardware status.

- Requisition relating Inventory and Employee to model the allocation and withdrawal of inventory items within the manufacturing system. Attributes are : DateTime, OrderNo, Amount and Location.

- Download relating NCPartProg and Controller / HanController objects, attributes being mainly time and date.

\section{Services and Communication}

Services related to Product objects can be noticed in the class boxes in Figure 3(a)-(c), e.g. :

- Assemble Product, inspect TestProduct, maintain McToolHard, setup NcMcTool, loadProg Controller : typical execution type of services.

- Check capacity : attached to AssgnJob object it represents an algorithmic check of availability of shop-floor capacity before assigning a job.

- CalculateStockLevel Inventory : typical calculation type of service.

Message connections are illustrated by referring to McBatchPart, see Figure 3. Processing progress is recorded within the object by the different states of the dynamic attribute CurrentStatus. This needs to be accessed, access being initiated from within the object StatusProgress by the service CheckProgress. The service CheckStatus is then activated within the object AccessStatus which sends a message to McBatchPart activating the service CalcProgress. This service calculates the current position / operation of the batch within the system and sends the result back to AccessStatus via the message connection. The result is then displayed, recorded or printed out using the service DisplayStatus.

\section{DISCUSSION}

In a manufacturing systems context Object Orientation has the potential to model data, knowledge, hardware, information, communication and events as separate objects. In effect these constitute the structural, transformation and procedural definition of a manufacturing system. In addition, objects which are found in one manufacturing system are very likely to occur in other Manufacturing systems with little or no modification. These objects and their relevant structures can be directly re-used, making it ideal to achieve the level of abstraction necessary for the production of reference models. 
Note that no subject of the model can be completely self-contained. There will always be instance connections and messages with objects belonging to other subjects. Sometimes it is expected that for some of the classes it will not be clear to which subject they belong.

Time sequences of events are not conveniently shown, certainly as far as visual interpretation of the model is concerned. This is an inherent weakness of OOA methodology and could be cured by either adopting a complementary methodology such as Activity Cycle Diagrams or by suitably modifying OOA, e.g. through definition of special types of services and special notation for message connections implementing event sequences.

Coad-Yourdon's OOA has already been proposed as a modelling methodology for manufacturing systems, albeit not from a reference standpoint. However, it was slightly modified and unnecessarily complicated into a 'new' methodology named HOOMA (Wu, 1995). The differences are first that HOOMA starts with a functional decomposition of the system in order to define in essence modelling domains. Then, functional decomposition in the form of Subsystem Relationship diagrams is carried out further before class and object definition. Objects and classes are distinguished into clients and servers, the distinction having though no apparent subsequent use. In HOOMA's whole-part structures parent objects cannot exist at the same time as their parts (children). This restriction seems to wipe out any local knowledge of system structure. State Transition Charts and Activity Cycle Diagrams are used mainly for improving ease of derivation of the correct instance and message connections.

As far as a comparison of OOA with CIM-OSA is concerned, it was noted (Nager, 1995) that CIMOSA leads to complicated lengthy models, compared to SADT and to Rumbaugh's Object Modelling Technique. $3 \times 3 \times 4$ types of CIM-OSA models are potentially needed for comprehensive representation of a manufacturing scenario. However, it must be recognised that several of these types are not straightforward to represent using other methodologies. In brief, it can be stated that CIM-OSA is just a modelling framework providing tools and methodology for modelling an enterprise or parts of it. Instanced models are not available and it is up to the users to develop them by plugging relevant knowledge into the modelling shells, as was done, for instance with respect to a CAD / PPS interface applicable to electromechanical product manufacturers (Nager, 1995).

The phenomenological view of the shop-floor model is merely a description of what should happen in a 'typical batch processing shop floor'. As such it could be termed an 'execution' View. There will certainly be applications referring to the Shop Floor with a different flavour, e.g. design, performance evaluation etc.

Each of these applications will make use of the objects, structures etc. contained in the phenomenological View of the model, but will need to extend it by adding further elements (objects, instance connections, messages, services etc.). Thus, the phenomenological View is also the 'core' View around which further extensions can be built to construct further Views. It should be noted that the phenomenological View needs to be generic enough to allow any additional View to be built on top of it. A preliminary discussion of such Views follows.

Specification of Shop Floor control : This View requires the existence of a controller hierarchy in the model for short term planning and monitoring of execution of production plans. Control components would need to be specified in terms of their function, status, and interaction. These would communicate with dynamic attributes of specific objects of the 'core' View, for instance via event-remembered type of objects.

Facility layout planning : This View would require adding e.g. location co-ordinates and perhaps location preferences / constraints to Hardware as well as demand indicators on top of the existing group technology codes to McBatchPart. The decision scheme (minimum cost equation, rules etc.) could be encapsulated into the additional object Layout.

Specification of machines and computer systems : Physical implementation can be achieved in many ways, requiring breaking down abstract objects into a set of physically implementable ones. The corresponding View would then be a 'concretisation' of the abstract 'core' View. 
Specification of interfaces with other manufacturing system areas : A number of reference models pertaining to individual manufacturing system areas may be constructed. These are expected to use many common objects, though not with a common set of attributes, providing a basis for integration. Integration should be enhanced by specifying explicit links through interface objects.

To illustrate the notion of collaborating views, an example of the possible role of the McToolHardware object in four views of a manufacturing system model is considered. The phenomenological view includes an attribute (Location) of the Machine object describing its location in a 3D space grid. In a layout design view a procedure (Optimise_location) is required to calculate the optimum location of the machine. In a physical element specification view instancing of the Machine object will depend on the technological characteristics derived after observation of all available instances of a Process_Plan class, hence there is a connection (both instance and message) to this object. In an interface specification view a subset of attributes of the Machine object can be identified for communication with other subjects, e.g. Production_Planning. For instance the Location attribute may be required by a service (Assign_LeadTime) calculating transportation time as part of the production lead time. This service may be attached to a Schedule object and effect its LeadTime attribute.

The model presented in Figure 3 can be seen as a generic model at the highest level of abstaction. There are, however, certain classes that have been expanded into more specific classes, such as the Toollnventory class. The CutTool subclass needed then to be related to ToolMag (and ToolHolder) classes with new instance connections which could not be applied to the parent class Inventory, covering diverse inventory items. It could, though, be related at the other end to class NcMcToolHardware because of its special one-to-one whole-part relationship with class ToolMag. These 'expansion' classes could have been omitted in the first place, with loss of all relevant attributes and relationships, and added at a later stage, observing the transformation rules mentioned above.

Further work is needed towards a structured abstraction of a manufacturing system by grouping objects into pre-defined generic classes such as : Information, Processors, Planners, Controllers, Calculators, Materials, Interfaces. In addition, the shop-floor model presented above needs further validation in order to become a true reference model, since this was based on own experience and academic material. Given the size of this task, it is no coincidence that no standardisation of any kind exists in the domain of batch manufacturing industries for mechanical engineering components.

\section{6}

\section{CONCLUDING REMARKS}

This work explores concepts relating to building reference models for integrated manufacturing systems. Object Orientation is advocated as a suitable paradigm for expressing such models and the application of Coad and Yourdon's Object Oriented Analysis is shown to be an adequate tool for this task. In particular, it is advocated that the OOA method in its pure form is better than any method based on it with a few 'enhancements' and modifications, since apart from other reasons, it retains the possibility for creating 'executable' models, i.e. a direct channel to software development based on the model.

The Shop-Floor model constructed using OOA can be seen as a 'core' model which can be used as basis for building application-specific Views relating to the same domain. Categorising the types of entities encountered in this domain, i.e. raising the level of abstraction, should provide for a modular approach to building 'custom' Views. Further work is being carried out in this direction.

\section{ACKNOWLEDGMENT}

Mark Williams acknowledges the financial support of the Engineering and Physical Sciences Research Council of Britain. 
Aguiar, C.W. and Weston, R.H. (1995) A model-driven approach to enterprise integration, International Journal of Computer Integrated Manufacturing, 8(3), 210-224.

Biemans, F.P.M. and Vissers, C.A. (1991) A Systems Theoretic View of Computer Integrated Manufacturing, International Journal of Production Research, 29(5), 947-966.

Bohms, H.M. and Tolman, F.P. (1993) A methodology for expressing CIM Reference Architectures, Robotics and Computer Integrated Manufacturing, 10(1/2), 131-140.

Burbidge, J.L., Flaster, P., Riis,J.O. and Svendsen, O.M. (1987) Integration in Manufacturing, Computers in Industry, 9, 297-305.

Camarinha-Matos, L.M., Pinheiro-Pitas, H., Rabelo, R. and Barata, J. (1994) Towards a taxonomy of CIM activities, International Journal of Computer Integrated Manufacturing, 8(3), 160-176.

Coad, P. and Yourdon, E. (1991) Object-Oriented Analysis, Prentice Hall.

Filip, F.G. and Neagu, G. : CIM in continuous and discrete manufacturing (1993) Object-Oriented generic modelling, Control Engineering Practice, 1(5), 815-825.

Hitomi, K. (1990) Manufacturing systems engineering : the concept, its context and the state of the art, International Journal of Computer Integrated Manufacturing, 3(5), 275-288.

Jochem, R., Rabe, M. and Sussenguth, W. (1989) An Object Oriented Analysis and Design Methodology for CIM systems, TOOLS'89, 75-84.

Krni, R., Rabinovitz, J. (1995) A taxonomy for the mateirals handling and tranfer environment, International Journal of Computer Integrated Manufacturing, 8(3), 177-188.

Lhoste, P., Jung, B., Mayer, F. and Morel, G. (1993) Reference modelling for distributed intelligent control system, Proceedings IEEE International Conference. on Systems, Man and Cybernetics, vol. 3, 84-89.

McCarthy, J.J. (1990) The CIM reference model as a tool for plant information and control systems development, Proc. of Advances in Instrumentation, 45 (3), ISA, 1455-1466.

Nager, N., Kapp, K.H., Schreiber, M. and Weber, U. (1995) Structuring and configuration of CIM systems for branch-specific medium-sized enterprises, Journal of Intelligent Manufacturing, 6, 191201.

$\mathrm{Wu}$, B. (1995) Object-oriented systems analysis and definition of manufacturing operations, International Journal of Production Research, 33(4), pp. 955-974.

\section{AUTHOR BIOGRAPHIES}

George Vosniakos graduated from the National Technical University of Athens in 1986. He obtained MSc and PhD degrees from UMIST in 1987 and 1991 respectively for work on CAE. He then worked as a Research Manager for a german CIM software house and in 1993 he joined UMIST as a lecturer in Manufacturing Systems. In 1995 he was elected Assistant Professor in Manufacturing at NTU Athens. $\mathrm{He}$ is the author of 22 articles on CAE topics.

Mark Williams graduated from Trent Polytechnic in 1992, having worked for GEC - Turbine Division for about 3 years. He, then, obtained an MSc in Advanced Manufacturing Technology and Systems Management at UMIST. Since 1994 he has been carrying out work on Business Process Re-engineering towards a PhD degree at UMIST. 